\title{
Chiral tensors of mixed Young symmetry
}

\author{
Marc Henneaux, Victor Lekeu, and Amaury Leonard \\ Université Libre de Bruxelles and International Solvay Institutes, \\ ULB-Campus Plaine CP231, B-1050 Brussels, Belgium \\ (Received 18 December 2016; published 24 April 2017)
}

\begin{abstract}
Chiral tensors of mixed Young symmetry, which exist in the same spacetime dimensions $2+4 n$ where chiral $p$ forms can be defined, are investigated. Such chiral tensors have been argued to play a central role in exotic formulations of gravity in six dimensions and possess intriguing properties. A variational principle that yields the chiral equations of motion is explicitly constructed and related to the action for a nonchiral tensor. The use of prepotentials turns out to be essential in our analysis. We also comment on dimensional reduction.
\end{abstract}

DOI: $10.1103 /$ PhysRevD.95.084040

\section{INTRODUCTION}

Chiral $p$ forms are an essential building block of various supergravity models [1]. These forms are defined to be such that their curvature $F$ is self-dual, $F={ }^{*} F$ [2]. In Minkowski space, chiral $p$ forms exist in dimensions $D=$ $2+4 n(n=0,1,2, \cdots)$ with $p=2 n$, since both $F$ and ${ }^{*} F$ must be forms of the same rank and the Hodge duality operation must square to the identity when acting on those $p$ forms, $\left({ }^{*}\right)^{2}=1$.

Following [3], an action principle that directly gives the chirality condition was constructed in [4]. This action principle not only automatically yields the chirality condition, which does not need to be separately imposed by hand, but it involves solely the $p$-form gauge potential without auxiliary fields, making the dynamics quite transparent. For instance, in the free case, it is just quadratic in the fields. One characteristic feature of the action of [4] is that it is not manifestly covariant in the sense that it is covariant, but in the sense that the fields do not transform off-shell in the standard way under Lorentz transformations. When couplings to gravity are included, this means that the fields do not transform in the standard way under spacetime diffeomorphisms. A similar feature (nonstandard transformations under spacetime diffeomorphisms) was encountered in the variational principle recently proposed in [5].

Tensor fields with mixed Young symmetry naturally appear in higher spacetime dimensions in the dual formulation of (linearized) gravity and in general studies of higher spin fields. They also appear in string field theory where they become massless in the tensionless limit.

Published by the American Physical Society under the terms of the Creative Commons Attribution 4.0 International license. Further distribution of this work must maintain attribution to the author(s) and the published article's title, journal citation, and DOI.
In the same spacetime dimensions $D=2+2 p$ ( $p$ even) where chiral $p$ forms exist, one can also consistently impose chirality conditions on the curvature tensor of mixed Young symmetry tensors when these are described by Young tableaux, the length of the first column of which is equal to $p$. This will be explained below. The $p$-form case corresponds to a Young tableau with a single column, the generalization considered here may involve an arbitrary number of columns.

Chirality conditions on mixed Young symmetry tensors have been actually considered in dimension six in the insightful and intriguing work [6-8], where it was argued that the strong coupling limit of theories having $N=8$ supergravity as their low energy effective theory in five spacetime dimensions should be a six-dimensional theory involving, besides chiral 2-forms, a chiral mixed $(2,2)$ tensor in place of the standard graviton. In $(2,2)$, the numbers give the number of boxes in the successive rows of the corresponding Young tableau. A very attractive feature of the six-dimensional theory containing the $(2,2)$ chiral tensor is that it provides a remarkable geometric interpretation of electric-magnetic gravitational duality in four dimensions.

Now, the discussion of [6-8] was performed at the level of the equations of motion. Although one may develop quantization methods that bypass the Lagrangian, the investigation of the quantum properties lies definitely on more familiar grounds when a self-contained action principle does exist.

The central result of our paper is to establish the existence of a local variational principle for free chiral tensors of mixed symmetry, which we explicitly write down. To achieve this goal, one must write the equations of motion in terms of prepotentials generalizing those introduced in [9]. Our approach relies on the tools for dealing with prepotentials and duality developed in $[10,11]$. As is the case for chiral $p$ forms, the action is not manifestly Lorentz invariant, even though the space of solutions is. 


\section{CHIRAL $(2,2)$ TENSOR IN $D=6$ SPACETIME DIMENSIONS}

\section{A. Equations of motion}

To illustrate the features brought in by tensors of mixed symmetry type, without having to deal with the extra technical complications of trace conditions, we concentrate on the simplest case, namely, tensors with $(2,2)$ Young symmetry in $D=6$ spacetime dimensions. This case is described by the Young tableau $\boxminus$. The general case will be discussed at the end of this letter. We denote the corresponding gauge field by $T_{\alpha_{1} \alpha_{2} \beta_{1} \beta_{2}}$, with $T_{\alpha_{1} \alpha_{2} \beta_{1} \beta_{2}}=$ $-T_{\alpha_{2} \alpha_{1} \beta_{1} \beta_{2}}=-T_{\alpha_{1} \alpha_{2} \beta_{2} \beta_{1}}$ and $T_{\left[\alpha_{1} \alpha_{2} \beta_{1}\right] \beta_{2}}=0$. The gauge symmetries are $\delta T_{\alpha_{1} \alpha_{2} \beta_{1} \beta_{2}}=\mathbb{P}_{(2,2)}\left(\partial_{\alpha_{1}} \eta_{\beta_{1} \beta_{2} \alpha_{2}}\right)$ where $\eta_{\beta_{1} \beta_{2} \alpha_{2}}$ is an arbitrary $(2,1)$ tensor. Here, $\mathbb{P}_{(2,2)}$ is the projector on the $(2,2)$ symmetry.

The gauge-invariant curvature, or "Riemann tensor," is a tensor of type $(2,2,2), \quad R \sim \boxminus$. In components, $R_{\alpha_{1} \alpha_{2} \alpha_{3} \beta_{1} \beta_{2} \beta_{3}}=\partial_{\left[\alpha_{1}\right.} T_{\left.\alpha_{2} \alpha_{3}\right]\left[\beta_{1} \beta_{2}, \beta_{3}\right]}$ and one has $R_{\alpha_{1} \alpha_{2} \alpha_{3} \beta_{1} \beta_{2} \beta_{3}}=$ $R_{\left[\alpha_{1} \alpha_{2} \alpha_{3}\right] \beta_{1} \beta_{2} \beta_{3}}=R_{\alpha_{1} \alpha_{2} \alpha_{3}\left[\beta_{1} \beta_{2} \beta_{3}\right]}$ as well as

$$
R_{\left[\alpha_{1} \alpha_{2} \alpha_{3} \beta_{1}\right] \beta_{2} \beta_{3}}=0 .
$$

The equations of motion for a general $(2,2)$ tensor express that the corresponding $(2,2)$ "Ricci tensor," i.e., the trace $R_{\alpha_{1} \alpha_{2} \beta_{1} \beta_{2}} \equiv R_{\alpha_{1} \alpha_{2} \alpha_{3} \beta_{1} \beta_{2} \beta_{3}} \eta^{\alpha_{3} \beta_{3}}$ of the Riemann tensor, vanishes,

$$
R_{\alpha_{1} \alpha_{2} \beta_{1} \beta_{2}}=0 \text {. }
$$

In six spacetime dimensions, the dual ${ }^{*} R$ of the Riemann tensor on, say, the first three indices ${ }^{*} R_{\alpha_{1} \alpha_{2} \alpha_{3} \beta_{1} \beta_{2} \beta_{3}}=$ $\frac{1}{3 !} \epsilon_{\alpha_{1} \alpha_{2} \alpha_{3} \lambda_{1} \lambda_{2} \lambda_{3}} R^{\lambda_{1} \lambda_{2} \lambda_{3}}{ }_{\beta_{1} \beta_{2} \beta_{3}}$ is traceless because of the cyclic identity (1), i.e.,

$$
{ }^{*} R_{\alpha_{1} \alpha_{2} \beta_{1} \beta_{2}}=0 .
$$

This implies that a $(2,2)$ tensor field $T$ with a self-dual or anti-self-dual Riemann tensor,

$$
R={ }^{*} R,
$$

(self-duality) or $R=-{ }^{*} R$ (anti-self-duality) is automatically a solution of the equations of motion (2). Note that this implies that ${ }^{*} R$ is also a $(2,2,2)$ tensor. The condition (4) is consistent because $\left({ }^{*}\right)^{2}=1$ in this case. The question addressed in this note is to derive (4) from a variational principle.

There is a mismatch between the number of Eqs. (4), namely 175 , and the number of components of the $(2,2)$ tensor field, namely, 105. But Eqs. (4) are not all independent. It is of course sufficient that the searchedfor variational principle yields a system of equations equivalent to (4).

\section{B. Electric and magnetic fields}

To identify such a subset derivable from a variational principle, we introduce the electric and magnetic fields. The electric field contains the components of the curvature tensor with the maximum number of indices equal to the time direction 0 , namely, two, $\mathcal{E}^{i j k l} \sim R^{0 i j 0 k l}$, or what is the same on-shell, the components of the curvature with no index equal to zero. Since in five dimensions, the curvature tensor $R_{\text {pijqkl }}$ is completely determined by the Einstein tensor $G^{i j}{ }_{k l}=\frac{1}{(3 !)^{2}} R^{a b c d e f} \varepsilon_{a b c}{ }^{i j} \varepsilon_{d e f k l}=R^{i j}{ }_{k l}-2 \delta_{[k}^{[i} R^{j]}{ }_{l]}+$ $\frac{1}{3} \delta_{[k}^{i} \delta_{l]}^{j} R$ (the Weyl tensor identically vanishes), one defines explicitly the electric field as

$$
\mathcal{E}^{i j k l} \equiv G^{i j k l} .
$$

Here, $R^{i j}{ }_{k l}=R^{m i j}{ }_{m k l}, R^{j}{ }_{l}=R^{m i j}{ }_{m i l}$ and $R=R^{m i j}{ }_{m i j}$ are the successive traces. Similar conventions will be adopted below for the traces of the tensors that appear. The electric field has the $(2,2)$ Young symmetry and is identically transverse, $\partial_{i} \mathcal{E}^{i j k l}=0$. It is also traceless on-shell,

$$
\mathcal{E}^{i k} \equiv \mathcal{E}^{i j k l} \delta_{j l}=0 .
$$

The magnetic field contains the components of the curvature tensor with only one index equal to 0 ,

$$
\mathcal{B}_{i j k l}=\frac{1}{3 !} R_{0 i j}{ }^{a b c} \varepsilon_{a b c k l} .
$$

It is identically traceless, $\mathcal{B}^{j l} \equiv \mathcal{B}^{i j k l} \delta_{i k}=0$, and transverse on the second pair of indices, $\partial_{k} \mathcal{B}^{i j k l}=0$. On-shell, it has the $(2,2)$ Young symmetry.

The self-duality equation (4) implies

$$
\mathcal{E}^{i j r s}-\mathcal{B}^{i j r s}=0 .
$$

Conversely, Eq. (8) implies all the components of the selfduality equation (4). This is verified in appendix A by repeating the argument of [12] given there for a (2) tensor, which is easily adapted to a $(2,2)$ tensor. We have thus replaced the self-duality conditions (4) by a smaller, equivalent, subset. One central feature of this subset is that it is expressed in terms of spatial objects.

Note that the trace condition (6) directly follows by taking the trace of (8) since the magnetic field is traceless. It appears as a constraint on the initial conditions because it does not involve the time derivatives of $T_{i j r s}$. There is no analogous constraint in the $p$-form case.

Since the number of components of the electric field is equal to the number of spatial components $T_{i j r s}$ of the $(2,2)$ tensor $T_{\alpha \beta \lambda \mu}$, one might wonder whether Eqs. (8) can be derived from an action principle in which the basic variables would be the $T_{i j r s}$. This does not work, however. Indeed, while the electric field involves only the spatial 
components $T_{i j r s}$ of the gauge field, the magnetic field involves also the gauge component $T_{0 j r s}$, through an exterior derivative. One must therefore get rid of $T_{0 j r s}$.

To get equations that involve only the spatial components $T_{i j r s}$, we proceed as in the 2-form case and take the curl of (8), i.e.

$$
\epsilon^{m n i j k} \partial_{k}\left(\mathcal{E}_{i j}{ }^{r s}-\mathcal{B}_{i j}{ }^{r s}\right)=0,
$$

eliminating thereby the gauge components $T_{0 j r s}$. We also retain Eq. (6), which is a consequence of (8) involving only the electric field. There is no loss of physical information in going from (8) to the system (6), (9). Indeed, as shown in appendix A, if (6) and (9) are fulfilled, one recovers (8) up to a term that can be absorbed in a redefinition of $T_{0 j r s}$. The use of (6) is crucial in the argument. It is in the form (6), (9) that the self-duality equations can be derived from a variational principle.

\section{Prepotentials-Action}

To achieve the goal of constructing the action for the chiral tensor, we first solve the constraint (6) by introducing a prepotential $Z_{i j r s}$ for $T_{i j r s}$. Prepotentials were defined for gravity in [4] and generalized to arbitrary symmetric tensor gauge fields in $[10,11]$. The introduction of a prepotential for the mixed tensor $T_{i j r s}$ proceeds along similar lines.

Explicitly, the prepotential $Z_{i j r s}$ provides a parametrization of the most general $(2,2)$ tensor field $T_{i j r s}$ that solves the constraint (6). One has

$$
T_{i j r s}=\mathbb{P}_{(2,2)}\left(\frac{1}{3 !} \epsilon_{i j}{ }^{k m n} \partial_{k} Z_{m n r s}\right)+\text { gauge transf, }
$$

which is a direct generalisation of the formula given in [9] for a (2) tensor. The prepotential is determined up to the gauge symmetries

$$
\delta Z_{i j r s}=\mathbb{P}_{(2,2)}\left(\partial_{i} \xi_{r s j}+\lambda_{i r} \delta_{j s}\right)
$$

where $\xi_{r s j}$ is a $(2,1)$ tensor parametrizing the "linearized spin-(2,2) diffeomorphisms" of the prepotential and $\lambda_{i r}$ a symmetric tensor parametrizing its "linearized spin- $(2,2)$ Weyl rescalings".

Because the Weyl tensor of a $(2,2)$ tensor identically vanishes, the relevant tensor that controls Weyl invariance is the "Cotton tensor," defined as

$$
D_{i j k l}=\frac{1}{3 !} \varepsilon_{i j a b c} \partial^{a} S_{k l}^{b c},
$$

where $\left.S^{i j}{ }_{k l}=G^{i j}{ }_{k l}-2 \delta_{[k}^{[i} G^{j]} l\right]+\frac{1}{3} \delta_{[k}^{i} \delta_{l]}^{j} G$ is the "Schouten tensor," which has the key property of transforming as $\delta S^{i j}{ }_{k l}=-\frac{4}{27} \partial^{[j} \partial_{[k} \lambda^{i]}{ }_{l]}$ under Weyl rescalings. The Cotton tensor $D_{i j k l}$ is a $(2,2)$ tensor which is gauge invariant under
(11), as well as identically transverse and traceless, $\partial_{i} D^{i j r s}=0=D^{i j r s} \delta_{j s}$. Furthermore, a necessary and sufficient condition for $Z_{i j r s}$ to be pure gauge is that its Cotton tensor vanishes.

The relation (10) implies that

$$
\mathcal{E}^{i j r s}[T[Z]] \equiv G^{i j r s}[T[Z]]=D^{i j r s}[Z] \text {. }
$$

The relation (10) gives the most general solution for $T_{i j r s}$ subject to the constraint that $\mathcal{E}^{i j r s}$ is traceless (this is proved in [10] for general higher spins described by completely symmetric tensors, and is easily extended to tensors with mixed Young symmetry). We note that in three dimensions, the analogous relations on the Cotton tensor for symmetric gauge fields have a nice supersymmetric interpretation [13]. It would be of interest to explore whether a similar interpretation holds here.

It follows from (10) that

$$
\frac{1}{2} \epsilon^{m n i j k} \partial_{k} \mathcal{B}_{i j}^{r s}=\dot{D}^{m n r s}[Z]
$$

and therefore, in terms of the prepotential $Z_{i j r s}$, the selfduality condition (9) reads

$$
\frac{1}{2} \epsilon^{m n i j k} \partial_{k} D_{i j}{ }^{r s}[Z]-\dot{D}^{m n r s}[Z]=0,
$$

an equation that we can rewrite as

$$
L^{m n r s \mid i j p q} Z_{i j p q}=0
$$

where the differential operator $L^{m n r s \mid i j p q}$ contains four derivatives and can easily be read off from (16). The operator $L^{m n r s \mid i j p q}$ is symmetric, so that one can form the action

$$
\begin{aligned}
S[Z] & =\frac{1}{2} \int d^{6} x Z_{m n r s}\left(L^{m n r s \mid i j p q} Z_{i j p q}\right) \\
& =\frac{1}{2} \int d^{6} x Z_{m n r s}\left(\dot{D}^{m n r s}[Z]-\frac{1}{2} \epsilon^{m n i j k} \partial_{k} D_{i j}{ }^{r s}[Z]\right),
\end{aligned}
$$

which yields (16) as equations of motion. Given that $Z \sim \partial^{-1} T$, this action contains the correct number of derivatives of $T$, namely two, and has therefore the correct dimension.

\section{Chiral and nonchiral actions}

The action (17) is our central result. Although not manifestly so, it is covariant. One way to see this is to observe that (17) can be derived from the manifestly covariant Curtright action for a $(2,2)$ field $[14,15]$ rewritten in Hamiltonian form. As explained in Appendix B, this 
action involves the spatial components $T_{i j r s}$ and their conjugate momenta $\pi^{i j r s}$ as canonically conjugate dynamical variables, while the temporal components $T_{0 i j k}$ and $T_{0 i 0 j}$ play the role of Lagrange multipliers for the "momentum constraint" $\mathcal{C}^{i j k} \equiv \partial_{l} \pi^{i j l k} \approx 0$ and the "Hamiltonian constraint" $\mathcal{C}^{i j} \equiv \mathcal{E}^{i k j}{ }_{k}[T] \approx 0$. These constraints can be solved by introducing two prepotentials $Z_{i j r s}^{(1)}$ and $Z_{i j r s}^{(2)}$. As for a chiral 2-form [16], the linear change of variables $\left(Z_{i j r s}^{(1)}, Z_{i j r s}^{(2)}\right) \rightarrow\left(Z_{i j r s}^{+}=Z_{i j r s}^{(1)}+Z_{i j r s}^{(2)}, Z_{i j r s}^{-}=Z_{i j r s}^{(1)}-Z_{i j r s}^{(2)}\right)$ splits the action as a sum of two independent terms, one for $Z_{i j r s}^{+}$and one for $Z_{i j r s}^{-}$. The Poincaré generators also split similarly, one for $Z_{i j r s}^{+}$and one for $Z_{i j r s}^{-}$, which transform separately. The action (17) is the action for $Z_{i j r s}^{+}$obtained though this decomposition procedure, with the identification $Z_{i j r s}^{+} \equiv Z_{i j r s}$. This second method for obtaining the action for a chiral $(2,2)$ tensor shows as a bonus how a nonchiral $(2,2)$ tensor dynamically splits as the sum of a chiral $(2,2)$ tensor and an antichiral $(2,2)$ tensor.

\section{E. Dimensional reduction}

Upon reduction from $5+1$ to $4+1$ dimensions, the prepotential $Z_{i j r s}$ decomposes into a $(2,2)$ tensor, a $(2,1)$ tensor and a (2) tensor. Using part of the Weyl symmetry, one can set the (2) tensor equal to zero, leaving one with a $(2,2)$ tensor and a $(2,1)$ tensor which are exactly the prepotentials of the pure Pauli-Fierz theory in $4+1$ dimensions [17], with the same action and gauge symmetries (see Appendix C). It is this remarkable connection between the (2,2)-self-dual theory in six spacetime dimensions and pure (linearized) gravity in five spacetime dimensions that is at the heart of the work [6,7]. We have shown here that the connection holds not just for the equations of motion, but also for the actions themselves.

\section{GENERALIZATIONS AND CONCLUSIONS}

The extension to more general two-column Young symmetry tensors is direct. The "critical dimensions" where one can impose self-duality conditions on the curvature are those where chiral $p$ forms exist. The first colum of the Young tableau characterizing the Young symmetry must have $p$ boxes, and the second column has then a number $q \leq p$ of boxes. So, in $D=6$ spacetime dimensions, one has also the interesting case of $(2,1)$ tensors, also considered in $[6,7]$. This case is treated along lines identical to those described here. For the next case $-D=10$ spacetime dimensions-, the first column must have length 4 , and the second column has length $q \leq 4$, an interesting example being the $(2,2,2,2)$ tensors. Again, the extension to this twocolumn symmetry case is direct, as in all higher spacetime dimensions $D=14,18,22,26, \ldots$.

The extension to more than two column Young symmetries is more subtle but proceeds as in [11], by relying on the crucial property demonstrated in [18], where it was shown that the second-order Fronsdal-Crurtright type equations can be replaced by equations on the curvatures, which involves higher order derivatives. The self-duality conditions can then be derived from an action principle involving the appropriate prepotentials. The action is obtained by combining the above derivation with the methods of [11] for introducing prepotentials.

The present analysis can be developed in various directions. First, following [6,7], it would be of great interest to consider the supersymmetric extensions of the six-dimensional chiral theory and to determine how the fermionic prepotentials enter the picture [19]. The attractive $(4,0)$-theory of $[6,7]$ deserves a particular effort in this respect [20]. Second, the inclusion of sources, which would be dyonic by the self-duality condition, and the study of the corresponding quantization conditions, would also be worth understanding [21-23].

Finally, we note that we restricted the analysis to flat Minkowski space. The trivial topology of $\mathbb{R}^{n}$ enabled us to integrate the differential equations for the prepotentials without encountering obstructions, using the Poincaré lemma of [24]. The consideration of Minkowski space is not optional at this stage since the coupling of a single higher spin field to curved backgrounds is problematic. It is known how to surpass the problems only in the context of the Vasiliev theory, which requires an infinite number of fields [25-29]. Important ingredients to extend the analysis of the present article to nonlinear backgrounds are expected to include the cohomological considerations of [16], the nonlinear extension of the higher spin Cotton tensors [30], as well as duality in cosmological backgrounds [31,32].

\section{ACKNOWLEDGMENTS}

We thank Xavier Bekaert and Andrea Campoleoni for useful discussions. V. L. and A. L. are Research Fellows at the Belgian F.R.S.-FNRS. This work was partially supported by the ERC Advanced Grant "High-Spin-Grav," by FNRS-Belgium (convention FRFC PDR T.1025.14 and convention IISN 4.4503.15) and by the "Communauté Française de Belgique" through the Action de Recherche Concertée (ARC) program.

\section{APPENDIX A: EQUATIONS OF MOTION}

In this appendix, we show the equivalences between the different forms of the self-duality equations given in the main text., $(4) \Leftrightarrow(8) \Leftrightarrow(6),(9)$.

$(4) \Leftrightarrow(8)$ : In components, the self-duality equation $R={ }^{*} R$ reads

$$
\begin{aligned}
& R_{0 i j k l m}=\frac{1}{3 !} \varepsilon_{i j}^{a b c} R_{a b c k l m} \\
& R_{0 i j 0 k l}=\frac{1}{3 !} \varepsilon_{i j}{ }^{a b c} R_{a b c 0 k l} .
\end{aligned}
$$


The first of these equations is equivalent to (8) by dualizing on the $\mathrm{klm}$ indices. Conversely, we must show that (8) implies (A2) or, equivalently, that (A1) implies (A2). To do so, we use the Bianchi identity $\partial_{\left[\alpha_{1}\right.} R_{\left.\alpha_{2} \alpha_{3} \alpha_{4}\right] \beta_{1} \beta_{2} \beta_{3}}=0$ on the curvature, which imples

$$
\partial_{0} R_{i j k \beta_{1} \beta_{2} \beta_{3}}=3 \partial_{[i} R_{j k] 0 \beta_{1} \beta_{2} \beta_{3}} .
$$

Therefore, taking the time derivative of equation (A1) gives

$$
\partial_{[k} R_{l m] 00 i j}=\frac{1}{3 !} \partial_{[k} R_{l m] 0 a b c} \varepsilon_{i j}{ }^{a b c},
$$

which is exactly the curl of (A2). Now, the tensor $R_{0 l m 0 i j}$ has the $(2,2)$ symmetry, and so does $\frac{1}{3 !} R_{0 l m a b c} \varepsilon_{i j}{ }^{a b c}=\mathcal{B}_{l m i j}$ because of equation (8) and the fact that $\mathcal{E}$ has the $(2,2)$ symmetry. Using the Poincaré lemma of [24] for rectangular Young tableaux, one recovers equation (A2) up to a term of the form $\partial_{[i} N_{j][k, l]}$ for $N_{j k}$ symmetric. This term can be absorbed in a redefinition of the $T_{0 j 0 k}$ components appearing in $R_{0 i j 0 k l}$. (In fact, the components $T_{0 j 0 k}$ drop from equation (A4), and this explains how one can get equation (A2) from (A1), which does not contain $T_{0 j 0 k}$ either.)

$(8) \Leftrightarrow(6),(9)$ : Equation (8) obviously implies (9). It also implies (6) because the magnetic field $\mathcal{B}$ is identically traceless. To prove the converse, we introduce the tensor $K_{i j k l m}=\varepsilon_{i j k}^{a b}(\mathcal{E}-\mathcal{B})_{l m a b}$. Equation (6) and the fact that $\mathcal{B}$ is traceless imply that $K$ has the $(2,2,1)$ symmetry, $K \sim \bigoplus$. Equation (9) states that the curl of $K$ on its second group of indices vanishes, $K_{i j k[m, n]}=0$. The explicit formula

$$
K_{i j k l m}=\frac{1}{3}\left(\varepsilon_{l m p q r} \partial^{p} T_{[i j, k]}^{q r}-\partial_{[0} T_{l m][i j, k]}\right)
$$

shows that the curl of $K$ on its first group of indices also vanishes, $\partial_{[i} K_{j k l] m n}=0$. Using the generalized Poincaré lemma of [33] for arbitrary Young tableaux, this implies that $K_{i j k l m}=\partial_{[i} \lambda_{j k][l, m]}$, where $\lambda_{j k l}$ is a tensor with the $(2,1)$ symmetry that can be absorbed in a redefinition of $T_{0 i j k}$. (Similarly to the previous case, those components actually drop from (9).) One finally recovers equation (8) by dualizing again $K$ on its first group of indices.

\section{APPENDIX B: HAMILTONIAN FORMULATION}

The Lagrangian for a nonchiral $(2,2)$ tensor $T_{\mu \nu \rho \sigma}$ is given by [15]

$$
\mathcal{L}=-\frac{5}{2} \delta_{\nu_{1} \ldots \nu_{5}}^{\mu_{1} \ldots \mu_{5}} M^{\nu_{1} \nu_{2} \nu_{3}}{ }_{\mu_{1} \mu_{2}} M_{\mu_{3} \mu_{4} \mu_{5}}{ }^{\nu_{4} \nu_{5}},
$$

where $M_{\mu \nu \rho \sigma \tau}=\partial_{[\mu} T_{\nu \rho] \sigma \tau}$ and $\delta_{\nu_{1} \ldots \nu_{5}}^{\mu_{1} \ldots \mu_{5}}=\delta_{\left[\nu_{1}\right.}^{\mu_{1}} \cdots \delta_{\left.\nu_{5}\right]}^{\mu_{5}}$. The associated Hamiltonian action is
$S_{H}=\int d t d^{5} x\left(\pi_{i j k l} \dot{T}^{i j k l}-\mathcal{H}-n_{i j k} \mathcal{C}^{i j k}-n_{i j} \mathcal{C}^{i j}\right)$,

where the Hamiltonian is

$$
\begin{gathered}
\mathcal{H}=\mathcal{H}_{\pi}+\mathcal{H}_{T} \\
\mathcal{H}_{\pi}=3\left(\pi^{i j k l} \pi_{i j k l}-2 \pi^{i j} \pi_{i j}+\frac{1}{3} \pi^{2}\right) \\
\mathcal{H}_{T}=\frac{5}{2} \delta_{j_{1} \ldots j_{5}}^{i_{1} \ldots i_{5}} M^{j_{1} j_{2} j_{3}}{ }_{i_{1} i_{2}} M_{i_{3} i_{4} i_{5}} j_{4} j_{5}
\end{gathered}
$$

The components $n_{i j k}=-4 T_{i j 0 k}$ and $n_{i j}=6 T_{0 i 0 j}$ of $T$ with some indices equal to zero only appear as Lagrange multipliers for the constraints

$$
\begin{gathered}
\mathcal{C}^{i j k} \equiv \partial_{l} \pi^{i j l k}=0 \\
\mathcal{C}^{i j} \equiv \mathcal{E}^{i k j}{ }_{k}[T]=0 .
\end{gathered}
$$

Those constraints are solved by introducing two prepotentials $Z_{i j k l}^{(1)}$ and $Z_{i j k l}^{(2)}$ through

$$
\begin{gathered}
\pi^{i j k l}=G^{i j k l}\left[Z^{(1)}\right] \\
T_{i j k l}=\frac{1}{3} \mathbb{P}_{(2,2)}\left(\epsilon_{i j}{ }^{a b c} \partial_{a} Z_{b c k l}^{(2)}\right) .
\end{gathered}
$$

In terms of prepotentials, we have up to a total derivative

$$
\begin{aligned}
& \pi_{i j k l} \dot{T}^{i j k l}=2 Z_{i j k l}^{(1)} \dot{D}^{i j k l}\left[Z^{(2)}\right] \\
& \mathcal{H}_{\pi}=3 G_{i j k l}\left[Z^{(1)}\right] S^{i j k l}\left[Z^{(1)}\right] \\
& \mathcal{H}_{T}=3 G_{i j k l}\left[Z^{(2)}\right] S^{i j k l}\left[Z^{(2)}\right] .
\end{aligned}
$$

Again, up to a total derivative, one has $G_{i j k l}[Z] S^{i j k l}[Z]=$ $\frac{1}{3 !} Z_{i j k l} \epsilon^{i j a b c} \partial_{a} D_{b c}{ }^{k l}[Z]$. Therefore, defining the prepotentials $Z_{i j k l}^{ \pm}=Z_{i j k l}^{(1)} \pm Z_{i j k l}^{(2)}$, the action splits into two parts, $S\left[Z^{+}, Z^{-}\right]=S^{+}\left[Z^{+}\right]-S^{-}\left[Z^{-}\right]$. The action $S^{+}\left[Z^{+}\right]$is exactly the action (17) provided in the text for a chiral tensor, while $S^{-}\left[Z^{-}\right]$is the analog action for an antichiral tensor (which differs from Eq. (17) only by the sign of the second term).

\section{APPENDIX C: DIMENSIONAL REDUCTION}

The prepotential decomposes into three tensors:

$$
Z_{I J K L} \rightarrow Z_{i j k l}, Z_{i j k 5}, Z_{i 5 j 5} .
$$

(For the purposes of this appendix, uppercase indices run from 1 to 5 , while lowercase indices run from 1 to 4 .) The $(2,2)$ tensor and the $(2,1)$ tensor are identified with the two 
prepotentials $P_{i j k l}$ and $\Phi_{i j k}$ for linearized gravity in $4+1$ dimensions [17] as

$$
Z_{i j k l}=12 \sqrt{3} P_{i j k l}, \quad Z_{i j k 5}=-3 \sqrt{3} \Phi_{i j k} .
$$

The (2) tensor $Z_{i 5 j 5}$ transforms under the Weyl symmetries (11) as $\delta Z_{i 5 j 5}=\frac{1}{3}\left(\lambda_{i j}+\delta_{i j} \lambda_{55}\right)$ and can, therefore, be set to zero. Remaining gauge transformations on $Z$ must respect this choice: this restricts the gauge parameters to $\lambda_{i j}=$ $-\delta_{i j} \lambda_{55}$ and $\xi_{i 55}=0$. The surviving gauge parameters are then $\lambda_{55}, \lambda_{i 5}, \xi_{i j k}$ and $\xi_{5 i j}$. (Note that $\xi_{i j 5}=-2 \xi_{5[i j]}$ is not independent due to the cyclic identity $\xi_{[I J K]}=0$.) The map with the gauge parameters of [17] is

$$
\begin{aligned}
\chi_{i j k} & =-\frac{\xi_{i j k}}{24 \sqrt{3}}, \quad S_{i j}=\frac{\xi_{5(i j)}}{12 \sqrt{3}}, \quad A_{i j}=-\frac{\xi_{5[i j]}}{12 \sqrt{3}} \\
\xi & =\frac{2 \lambda_{55}}{9 \sqrt{3}}, \quad B_{i}=\frac{2 \lambda_{i 5}}{9 \sqrt{3}} .
\end{aligned}
$$

This shows that field content and gauge symmetries match. For the comparison of the actions, one needs the following expressions for the reduction of the Cotton tensor:

$$
\begin{aligned}
D_{i j}{ }^{k l} & =-\frac{2}{\sqrt{3}} \varepsilon_{i j a b} \partial^{a}\left(E^{k l b}+\delta^{b[k} E^{l]}\right) \\
D_{i j}{ }^{k 5} & =2 \sqrt{3} \varepsilon_{i j a b} \partial^{a}\left(R^{k b}-\frac{1}{3} \delta^{k b} R\right) \\
D_{i j}{ }^{j 5} & =\frac{1}{\sqrt{3}} \varepsilon_{i a b c} \partial^{a}\left(E^{b c j}+\delta^{j b} E^{c}\right),
\end{aligned}
$$

where $R^{i j}[P]$ and $E^{i j k}[\Phi]$ are defined as

$$
\begin{aligned}
& R^{i j}[P]=\frac{1}{(3 !)^{2}} \varepsilon^{i a b c} \varepsilon^{j d e f} \partial_{a} \partial_{f} P_{b c d e} \\
& E^{i j k}[\Phi]=\frac{1}{2.3 !} \varepsilon^{i j d e} \varepsilon^{k a b c} \partial_{a} \partial_{e} \Phi_{b c d}
\end{aligned}
$$

and the traces are $R=R_{i}^{i}, E^{i}=E^{i j}{ }_{j}$. Using these formulas, one recovers the action of [17] for linearized gravity in $4+1$ dimensions in the prepotential formalism.
[1] N. Marcus and J. H. Schwarz, Phys. Lett. 115B, 111 (1982); J. H. Schwarz and P. C. West, Phys. Lett. 126B, 301 (1983); J. H. Schwarz, Nucl. Phys. B226, 269 (1983); P. S. Howe and P. C. West, Nucl. Phys. B238, 181 (1984); L. J. Romans, Nucl. Phys. B276, 71 (1986).

[2] Antichiral $p$ forms such as $F=-{ }^{*} F$ enjoy similar properties. For definiteness, we shall focus here on the chiral case.

[3] R. Floreanini and R. Jackiw, Phys. Rev. Lett. 59, 1873 (1987).

[4] M. Henneaux and C. Teitelboim, Phys. Lett. B 206, 650 (1988).

[5] A. Sen, J. High Energy Phys. 07 (2016) 017.

[6] C. M. Hull, Nucl. Phys. B583, 237 (2000).

[7] C. M. Hull, J. High Energy Phys. 12 (2000) 007.

[8] C. M. Hull, J. High Energy Phys. 09 (2001) 027.

[9] M. Henneaux and C. Teitelboim, Phys. Rev. D 71, 024018 (2005).

[10] M. Henneaux, S. Hörtner, and A. Leonard, J. High Energy Phys. 01 (2016) 073.

[11] M. Henneaux, S. Hörtner, and A. Leonard, Phys. Rev. D 94, 105027 (2016).

[12] C. Bunster, M. Henneaux, and S. Hortner, J. Phys. A 46, 214016 (2013); 46, 269501(E) (2013).

[13] S. M. Kuzenko and D. X. Ogburn, Phys. Rev. D 94, 106010 (2016); S. M. Kuzenko, Phys. Lett. B 763, 308 (2016); S. M. Kuzenko and M. Tsulaia, Nucl. Phys. B914, 160 (2017).

[14] T. Curtright, Phys. Lett. B 165, 304 (1985).

[15] N. Boulanger and S. Cnockaert, J. High Energy Phys. 03 (2004) 031.
[16] X. Bekaert and M. Henneaux, Int. J. Theor. Phys. 38, 1161 (1999).

[17] C. Bunster, M. Henneaux, and S. Hörtner, Phys. Rev. D 88, 064032 (2013).

[18] X. Bekaert and N. Boulanger, Phys. Lett. B 561, 183 (2003).

[19] C. Bunster and M. Henneaux, Phys. Rev. D 86, 065018 (2012); C. Bunster, M. Henneaux, S. Hörtner, and A. Leonard, Phys. Rev. D 90, 045029 (2014).

[20] M. Henneaux, V. Lekeu, and A. Leonard (to be published).

[21] S. Deser, A. Gomberoff, M. Henneaux, and C. Teitelboim, Phys. Lett. B 400, 80 (1997); Nucl. Phys. B520, 179 (1998).

[22] N. Seiberg and W. Taylor, J. High Energy Phys. 06 (2011) 001.

[23] C. Bunster and M. Henneaux, Phys. Rev. D 88, 085002 (2013).

[24] M. Dubois-Violette and M. Henneaux, Lett. Math. Phys. 49, 245 (1999); Commun. Math. Phys. 226, 393 (2002).

[25] M. A. Vasiliev, Int. J. Mod. Phys. D 05, 763 (1996).

[26] M. A. Vasiliev, C.R. Phys. 5, 1101 (2004).

[27] X. Bekaert, N. Boulanger, and P. Sundell, Rev. Mod. Phys. 84, 987 (2012)

[28] V. E. Didenko and E. D. Skvortsov, arXiv:1401.2975.

[29] R. R. Metsaev, Phys. Lett. B 309, 39 (1993).

[30] H. Linander and B. E. W. Nilsson, J. High Energy Phys. 07 (2016) 024.

[31] B. Julia, J. Levie, and S. Ray, J. High Energy Phys. 11 (2005) 025; B. L. Julia, arXiv:hep-th/0512320.

[32] S. Hörtner, Phys. Rev. D 95, 024039 (2017).

[33] X. Bekaert and N. Boulanger, Commun. Math. Phys. 245, 27 (2004). 